\title{
A WSN-Based Intrusion Alarm System to Improve Safety in Road Work Zones
}

\author{
Jose Martin, Alba Rozas, and Alvaro Araujo \\ Departamento de Ingeniería Electrónica, ETSI Telecomunicación, Universidad Politécnica de Madrid, 28040 Madrid, Spain
}

Correspondence should be addressed to Jose Martin; jmartin@die.upm.es

Received 19 February 2016; Revised 3 May 2016; Accepted 12 May 2016

Academic Editor: Jong-Jae Lee

Copyright (C) 2016 Jose Martin et al. This is an open access article distributed under the Creative Commons Attribution License, which permits unrestricted use, distribution, and reproduction in any medium, provided the original work is properly cited.

Road traffic accidents are one of the main causes of death and disability worldwide. Workers responsible for maintaining and repairing roadways are especially prone to suffer these events, given their exceptional exposure to traffic. Since these actuations usually coexist with regular traffic, an errant driver can easily intrude the work area and provoke a collision. Some authors have proposed mechanisms aimed at detecting breaches in the work zone perimeter and alerting workers, which are collectively called intrusion alarm systems. However, they have several limitations and have not yet fulfilled the necessities of these scenarios. In this paper, we propose a new intrusion alarm system based on a Wireless Sensor Network (WSN). Our system is comprised of two main elements: vehicle detectors that form a virtual barrier and detect perimeter breaches by means of an ultrasonic beam and individual warning devices that transmit alerts to the workers. All these elements have a wireless communication interface and form a network that covers the whole work area. This network is in charge of transmitting and routing the alarms and coordinates the behavior of the system. We have tested our solution under real conditions with satisfactory results.

\section{Introduction}

Each year, road accidents are the cause of an unacceptable number of fatalities and injuries everywhere in the world. As a result, road safety is one of the main concerns for citizens and governments nowadays and a lot of effort has been put into reducing these figures. For instance, road accidents and fatalities in the EU in 2015 have fallen by $22 \%$ and $46 \%$, respectively, since 2004, according to data published by the European Road Safety Observatory. Despite these encouraging results, there is still a lot of work ahead.

One of the main action points in reducing traffic accidents is improving the conditions of roads and other infrastructures. These actions usually encompass the deployment of construction sites on roads and highways. In many cases, the complete shutdown of the roadway is not possible and these work zones have to share the road surface with regular traffic, with little or no protection between them. This results in an evident hazardous environment for both workers and road users. A survey released by the UK Highways Agency in 2006 stated that up to $20 \%$ of road workers had suffered some injury caused by passing vehicles in the course of their careers and $54 \%$ had experienced a near miss with a vehicle.

In order to increase the visibility and safety of workers, construction sites are typically marked by signs, cones, and other channelizing devices, as seen in Figure 1. Their goal is to warn and guide road users creating a barrier around the perimeter of the work zone. A very important characteristic of these barriers is that they have to be composed of crashworthy devices, such as cones and barrels, in order to cause minimal damage if hit by a vehicle. However, this implies that distracted or errant drivers can easily intrude into the work area.

Over the years, several systems and methods have been proposed to address this problem, with the particular goal of alerting workers about the immediate danger. They are generally called intrusion alarm systems.

The work in [1] is the most recent survey on traffic safety devices, carried out by the Kansas Department of Transportation in 2011. In this work, intrusion alarm systems are described as any sensing technology mounted on work zone barriers that triggers an alarm in the event of an invasion 


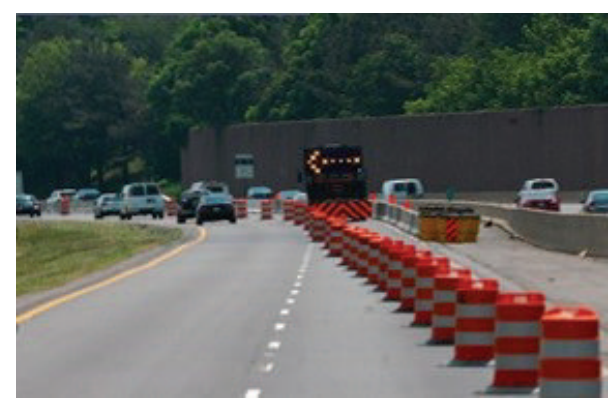

FIgURE 1: Example of a work zone delimited by barrels.

by an errant driver. Their main constraint is that the alarm has to warn workers effectively and with enough reaction time to avoid the incoming danger. The majority of systems are based on microwave and infrared beams, while others utilize pneumatic tubes placed on the ground. The study concludes that there are evident problems with the existing systems, based on surveys to users. They have a high number of false alarms, their setup process is long and difficult, and, more importantly, the generated alarms are very difficult or impossible to hear over the usual noise of work zones.

The work in [2] is another review of intrusion alarm systems published by the AHMCT Research Center in 2009. Apart from some of the systems already analyzed in [1], this paper reviews other types of devices. The first ones are systems that augment the traditional road cone with a tipping sensor that triggers an alarm when the cone is hit by a vehicle. The most prominent example of this kind of systems is the SonoBlaster, commercialized by Transpo Industries. These devices present the following problems: they can generate false positives if the cones are tipped by the wind or any other not threatening agent; it could be possible for a vehicle to invade the work area without tipping a cone; and the durability of the device could be compromised by the impacts.

They also review systems in which a transmitter unit and a receiver unit are aligned to create an invisible continuous barrier along the perimeter of the work zone based on a pulsed microwave beam [3]. The main disadvantage of this system is the complexity of its deployment and maintenance, since the alignment between the transmitter and the receiver has to be preserved at all times. Both these devices have another shared drawback, present in most of the analyzed intrusion systems: the alarm that the systems generate is very difficult to hear in a construction environment.

To address this problem, a device called Wireless Warning Shield was proposed and patented in 2006 [4]. It was based on a wireless communication system mounted on cones and also equipped with tipping sensors. Each individual worker would also wear an alert device, which would receive alarms from the system wirelessly in case of a breach. However, this product was never fully developed and never reached a functional stage.

The authors in [2] conclude that, given the virtually inexistent commercialization of these kind of devices, current intrusion alarm systems have significant limitations both from their technical implementation as well as from worker acceptance.

In a different line of research, several systems have been proposed within the field of traffic management and road safety that are based on Wireless Sensor Networks (WSNs) [5-7]. Over the last few years, WSNs have been applied to a huge variety of scenarios due to their low cost, high scalability, and ease of deployment. In this particular field, several works make use of WSNs to monitor, study, and evaluate traffic patterns using techniques based on infrared [8], ultrasonic [9], or magnetic sensing [10, 11]. In [12], the authors propose a WSN-based system especially aimed at monitoring traffic in or near short-term work zones. However, to the best of our knowledge, there are no existing systems that use WSNs in the context of intrusion detection. WSNs have the potential of enhancing these systems by providing a way of communication to work zone environments.

In this paper, we propose an intrusion alarm system for road work zones based on a WSN. Our system is composed of two main elements: vehicle detectors to monitor the perimeter and warning devices to individually alert the workers. All these elements are connected forming a network that covers the whole work area. Its main features are as follows:

(i) Individual alerts: workers are warned of the incoming danger by means of their individual warning device. This way we can ensure that workers are effectively alerted even if they are far from the source of the alarm or in a noisy environment, which was one of the most worrying problems of previous intrusion alarm systems. In addition, these devices can be used to monitor and control the working conditions, such as temperature and impacts. They are also light and comfortable to wear, guaranteeing worker acceptance.

(ii) Wireless Sensor Network: the WSN provides a way of communication within the work zone. Not only could WSNs reliably transmit and present alarms, but they could also be used to communicate any other type of information relevant to the particular scenario. For instance, the crew manager can monitor the activity and location of workers by means of their individual warning devices.

(iii) Robustness: vehicle detectors can detect invasions of the perimeter whether the cone is hit or not. This is an advantage over previous systems.

(iv) Easy deployment and setup: our vehicle detectors do not have separate transmitter and receiver units. Thus, there is no need for a precise alignment when deploying the system. They are also lightweight and easy to mount. This expedites the deployment and setup phase in comparison to most previous systems.

(v) Autonomy: all the elements are powered by rechargeable batteries, whose duration is completely adequate for regular work zone schedules. 


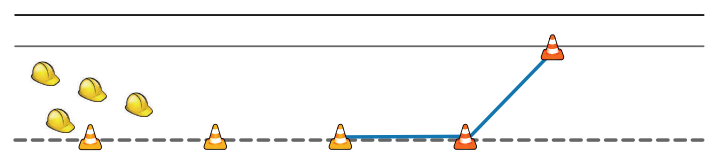

\& Common road cone
\& Cone equipped with vehicle detector
Worker

FIgURE 2: Top view of the target scenario.

\section{Materials and Methods}

Our intrusion alarm system is especially aimed at improving safety in short-term work zones. On these works, it is common for the workers to share the road with the adjacent traffic with not enough safety measures that protect them from errant drivers. The most common entry point into the work zone is the first part of the perimeter, which is usually delimited by cones, so these are the places where the vehicle detectors are placed (see Figure 2). The workers will be alerted by a personal warning device that all of them carry during their work activities. The vehicle sensors and warning devices are connected by a wireless network to deliver the alerts and configure the system.

In order to design our system, some key goals were defined to ensure the suitability to the target scenario:

(i) The deployment should be easy, allowing workers to setup the system in a reasonable time.

(ii) The system should be usable in the majority of the road scenarios, so there should not be special placement considerations that limit the usage scenario.

(iii) All devices should be battery-powered, since power is not usually available at these locations. The battery recharge/replacement should be easy enough to be done by people with no technical skills.

(iv) The warning devices will be worn continuously by the workers, so they should be comfortable to use and warn workers in a quick, safe, and reliable way that does not interfere with common road maintenance tasks.

(v) The total cost of the solution should be as moderate as possible to encourage its incorporation to the target scenarios.

2.1. Sensor Node. The sensor nodes are responsible for detecting vehicles breaching the perimeter of the work zone. They will be installed on cones or on any other barrier that delimits the work area. These nodes also have wireless networking capabilities to transmit the alarms and exchange and route the messages that the system uses to achieve its functionality. Optionally, they may include a siren to warn errant drivers and nearby workers.
The sensor node is a custom development built around an MSP430F249 microcontroller. This microcontroller has a low power consumption and all the required interfaces to communicate with the vehicle detector, the wireless transceiver, and other built-in modules, which can be seen in Figure 3.

The vehicle detection is achieved with the MaxBotix MB7076 ultrasonic range sensor. It was chosen because it provides an adequate measure rate needed to detect passing vehicles and its protective covering is convenient for its use in harsh construction zones.

This sensor has a digital data output that returns the distance to objects in its line of sight. At the system deployment, each sensor node is configured with a particular threshold distance. If any sensor node detects an object closer than that distance on its line of sight, it will generate an intrusion alert that will be sent to the workers and cause the siren (if present) to be activated.

Inside the sensor nodes, there are some other modules required for the application. Since they are powered from a LiPo battery, they include a common USB charger, a fuel gauge to manage low battery situations, and a power module to provide the necessary voltage levels in the different parts of the node. It also incorporates a MEMS-based accelerometer, used to detect impacts from errant vehicles in case they directly hit the cone or barrier on which the sensor is mounted, rather than trespassing on its virtual line of sight.

The network capabilities of the node are provided by a CC1101 wireless transceiver operating on the $868 \mathrm{MHz}$ Short Range Devices (SRD) band allocated by the European ETSI. The transceiver output is coupled to a PCB meander monopole antenna allowing a transmission power of up to $16 \mathrm{~mW}$. The radio module allows the node to communicate in point-to-point or point-to-multipoint configurations.

According to its own circumstances and the network conditions, each sensor has four possible working states:

(i) Power off: when the system is not deployed, the node remains in a soft power-off mode preventing battery drain.

(ii) Ready: at the system deployment stage, alarm events are not desired, so the sensor nodes maintain the network connection, but they do not transmit intrusion alerts to the warning devices.

(iii) Detection: this is the usual working state in which the nodes are actively monitoring their virtual barrier. If any of the sensor nodes detect any object in their defined line of sight, the alarm mode will be triggered.

(iv) Alarm: when a vehicle is detected, the sensor nodes wake up and activate the siren if present. This situation can be reverted from the crew manager's personal warning device.

The final node is enclosed in a protective casing as shown in Figure 4.

2.2. Warning Devices. These devices will be carried by each worker in the area and are in charge of receiving and presenting the danger alerts generated on the sensor nodes. 


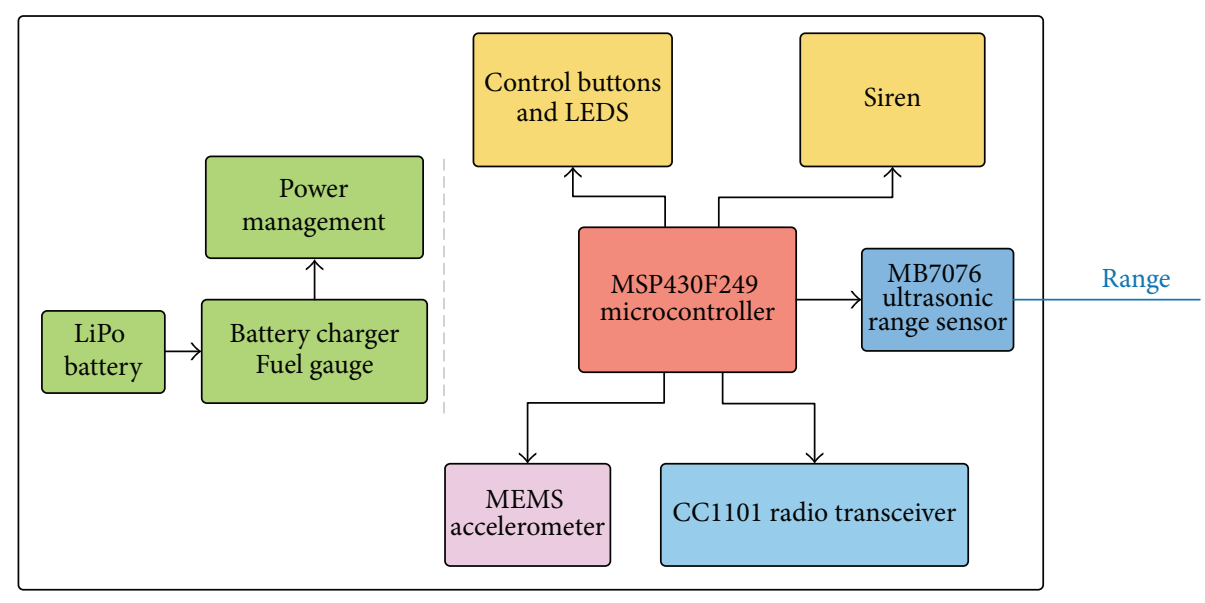

FIgURE 3: Sensor node hardware architecture.

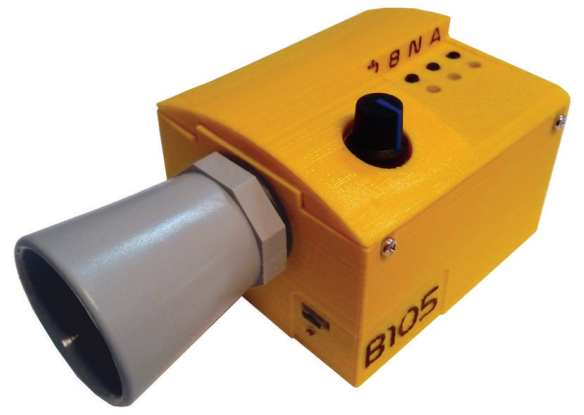

FIGURE 4: Sensor node.

Also, they can be used to check and manage the operating state of the system.

They have two software-defined configurations: master and normal. The warning devices configured as master are able to stop the alarm after a vehicle detection and revert the system to its monitoring state, while the normal nodes are limited to display danger warnings. The master warning device is meant to be carried by the crew manager or lead worker of the area.

Since this device has to be carried by the workers for the entire duration of their shift without affecting their usual activities, its main requirements are lightness, compactness, and comfort of use. Thus, a commercially available programmable watch was selected, the ez430-Chronos. In order for it to work as a warning device, some software was developed to communicate with the sensor nodes and present warnings or information to the user.

The ez430-Chronos watch (Figure 5) was developed by BM Innovations and it is offered by Texas Instruments as a complete development kit for its line of wireless System on Chip (SoC) solutions. The watch includes a CC430F6137 SoC which combines an MSP430 microcontroller and a CC1101based wireless transceiver. Along with it, the watch includes a 96-segment LCD screen and a piezo buzzer which are used to show the warnings to the workers.

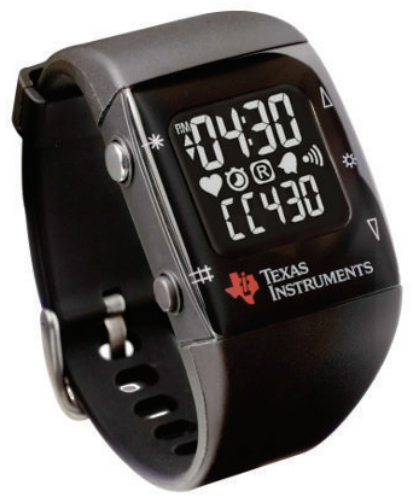

FIgURE 5: ez430-Chronos watch used as the warning device.

The wireless transceiver built inside the watch is used to communicate with the sensor nodes in the $868 \mathrm{MHz}$ band. This communication is based on a periodic polling scheme in order to save battery. The polling period is dynamically tuned according to the wireless link quality to ensure prompt warning reception.

2.3. Wireless Network. As stated previously, all the nodes in the system communicate using the $868 \mathrm{MHz}$ SRD band. This band was chosen because it provides an adequate range and it can be used without a license. The physical level is builtin on the CC1101 transceivers. Over that level, a wireless star topology has been developed, using one of the sensor nodes as the central hub. This topology was chosen in order to facilitate a coordinated behavior of the network. A sensor node was chosen for this role due to its higher battery capacity since the central hub's radio has to be continuously active. The logical connection of the nodes is represented in Figure 6.

The wireless network is used to synchronize the state of each sensor node and distribute the danger alarms that any of them may trigger. When an alarm is triggered, each node is informed so the warning devices will alert the worker and 


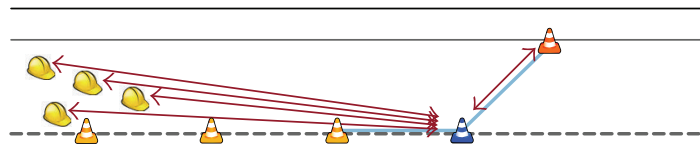

8 Sensor node (central hub)

\& Sensor node

C. Worker with warning device

FIGURE 6: Network topology at the target scenario.

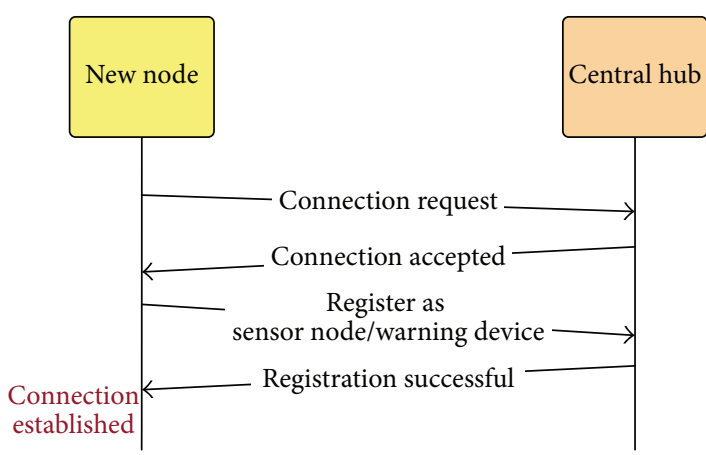

FIGURE 7: Message exchange on a new node registration.

the sensor nodes will activate their sirens (if present). The workers, upon hearing the alarm, should clear the road and look for any vehicle intrusion in the work zone. Once the situation is controlled, the worker that carries the master warning device can reset the alarm, turning off all the alerts, which will revert the system to the detection state.

The most important tasks carried out by the network are node registration, alarm triggering, and alarm reset. The message exchange that takes place across the network on these events is presented in the next subsections. Other minor network uses are the activation and deactivation of the system, the transmission of low battery alerts, and the centralized shutdown of all the nodes.

2.3.1. Node Registration. Since the system is going to be used in a wide spectrum of scenarios, the number of sensor nodes and warning devices can be very variable. Therefore, a dynamic device registration and deregistration protocol were implemented. The message exchange performed by the central hub and the new node is represented in Figure 7.

\subsubsection{Intrusion Alarm Triggering and Reset. When a sensor} node detects a vehicle, it communicates the event to the central hub, which instructs the remaining sensor nodes to activate their sirens. Since the warning devices follow a polling scheme and are not permanently connected, they will receive the alarm on their next connection to the central hub. The entire message exchange is shown in Figure 8.
TABLE 1: Speed effect on vehicle detection.

\begin{tabular}{lc}
\hline Distance from vehicle $(d)$ & $3 \mathrm{~m}$ \\
Angle $(\alpha)$ & $0^{\circ}$ \\
Ambient temperature & $18^{\circ} \mathrm{C}$ \\
Tested speeds $(\bar{v})$ & $30 \mathrm{~km} / \mathrm{h}, 50 \mathrm{~km} / \mathrm{h}, 70 \mathrm{~km} / \mathrm{h}, 90 \mathrm{~km} / \mathrm{h}$ \\
Repetitions (at each speed) & 10 \\
Results & Vehicle detected on all runs \\
\hline
\end{tabular}

After the work zone is considered safe again, the crew manager will issue an alarm reset. This procedure is similar to the alarm triggering event, with the only difference that the node starting the operation is the crew manager's warning device.

\section{Results and Discussion}

Various tests were performed to check the system functionality and its ability to detect and alert about work zone intrusions. Most of them were conducted on a closed track environment, where a typical short-term work zone scenario was recreated using cones. A sensor node was placed on top of a cone as can be seen in Figure 9.

The main variables that theoretically may affect the range measurement with an ultrasonic range sensor are the combination of the distance to the target, its speed, and the beam's angle of impact. The cone with the attached sensor node was carefully positioned on every test to analyze the impact of each of these three variables on the vehicle detection capabilities when a car enters the simulated work zone scenario. These parameters are shown in Figure 10.

The first test evaluated the effect of speed on vehicle detection. In this test, the car passed 3 meters away from the sensor node, whose line of sight was perpendicular to the road trajectory. Under these conditions, a speed from 30 to $90 \mathrm{~km} / \mathrm{h}$, with $20 \mathrm{~km} / \mathrm{h}$ increments, was tested. For each of these speeds, a total of 10 runs were evaluated. The results of this particular experiment can be seen in Table 1. On every run at each speed, the vehicle was correctly detected and the alarm was successfully sent to the warning devices, leading to the conclusion that reasonable car speeds do not cause a significant effect on vehicle detection.

The second test performed aimed to determine the relevance of the angle formed by the sensor beam and the vehicle trajectory. In this case, the distance was fixed to 3 meters in the perpendicular direction, and the speed was set to $60 \mathrm{~km} / \mathrm{h}$. Angles from $-60^{\circ}$ to $+60^{\circ}$ were tested, in $30^{\circ}$ increments, with 10 runs for each setup. The results of this evaluation can be seen in Table 2. As in the previous experiment, no detection misses were reported in the test, allowing us to conclude that the angle does not significantly affect the detection capability of the sensor.

The last road test involves the distance between the car and the sensor node. The speed was set to $60 \mathrm{~km} / \mathrm{h}$, and the angle was fixed at $0^{\circ}$; that is, the line of sight was perpendicular to the vehicle trajectory. For each distance, 10 vehicle runs were tested. The results of this experiment 


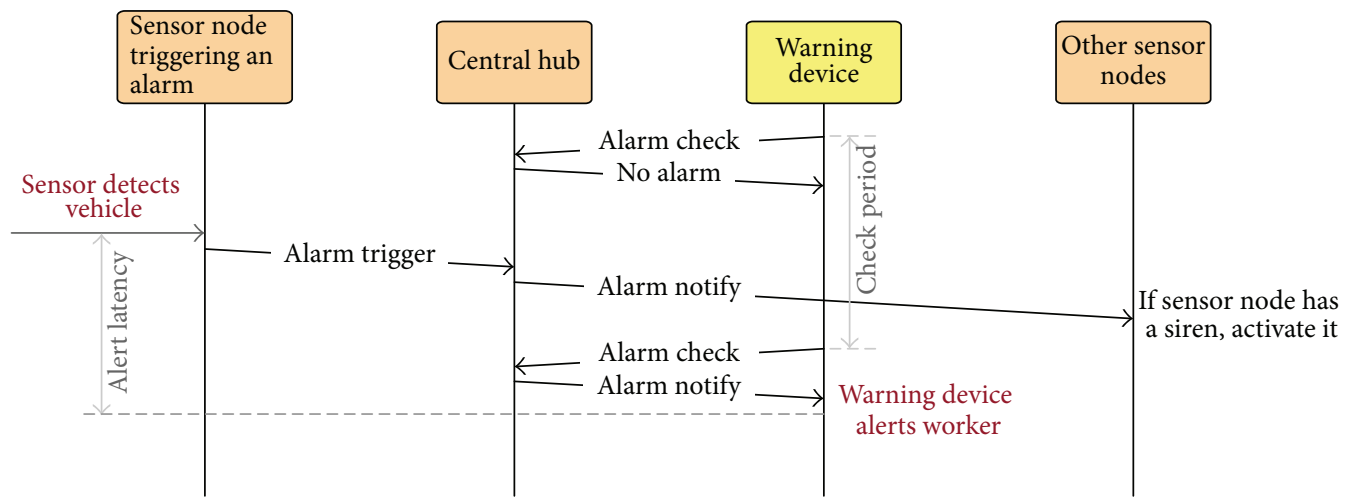

FIgURE 8: Message exchange when triggering an alarm.

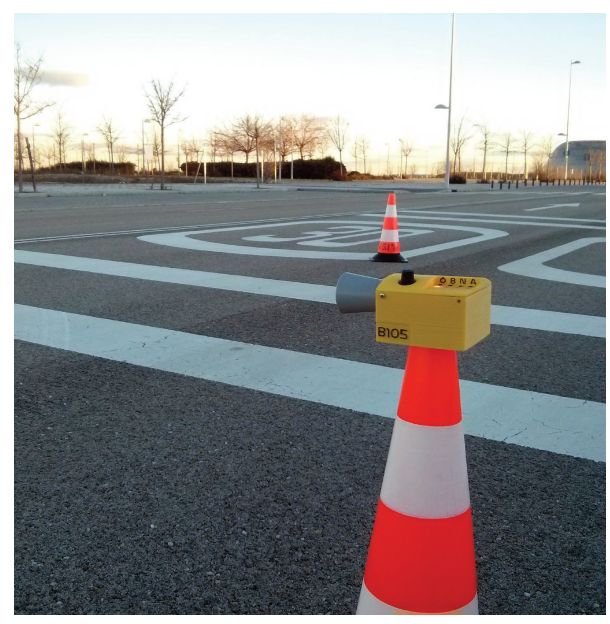

FIGURE 9: Sensor node mounted on a cone.
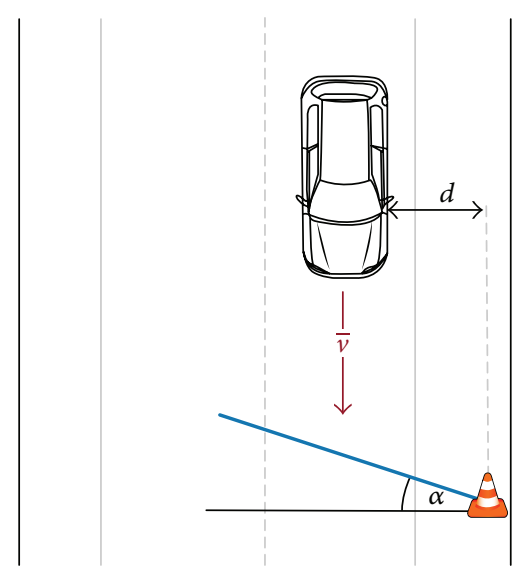

Figure 10: Parameters affecting vehicle detection.

are presented in Table 3 and Figure 11. A performance degradation can be seen on the 7-meter test which is probably caused by the short time that the car is situated in front of the sensor detection zone, aggravated by the longer distance which causes a lower return signal level.

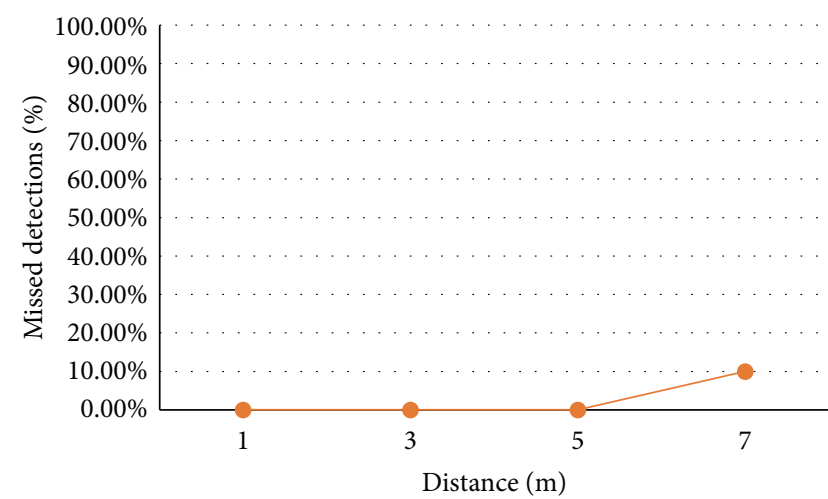

FIGURE 11: Undetected vehicles versus distance.

Table 2: Angle of incidence effect on vehicle detection.
Vehicle speed $(\bar{v})$

Distance from vehicle $(d)$

Ambient temperature

Tested angles $(\alpha)$

Repetitions (at each angle)

Results

\section{$60 \mathrm{~km} / \mathrm{h}$ \\ $3 \mathrm{~m}$}

$18^{\circ} \mathrm{C}$

$-60^{\circ},-30^{\circ}, 0^{\circ}, 30^{\circ}, 60^{\circ}$

10

Vehicle detected on all runs
TABLE 3: Distance effect on vehicle detection.
Vehicle speed $(\bar{v})$

Angle $(\alpha)$

Ambient temperature

Tested distances $(d)$

Repetitions (at each distance)

Results
$60 \mathrm{~km} / \mathrm{h}$

$0^{\circ}$

$18^{\circ} \mathrm{C}$

$1 \mathrm{~m}, 3 \mathrm{~m}, 5 \mathrm{~m}, 7 \mathrm{~m}$

10

Vehicle detected on all runs except for 2 times on $7 \mathrm{~m}$ test
The warning capability of the system as whole was evaluated in another series of tests. In them, the latency from the moment the vehicle crosses the virtual barrier to the moment the alarm is heard in the warning devices is measured. This parameter was evaluated in a series of experiments in which the distance between the central sensor node and a single 


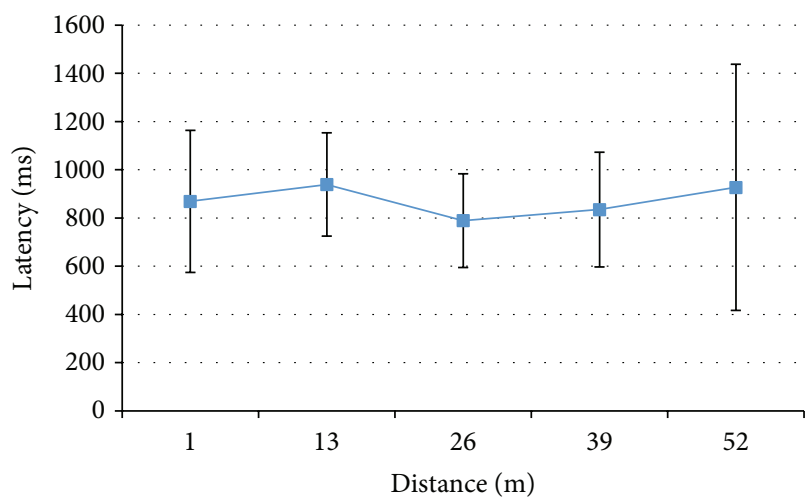

(a)

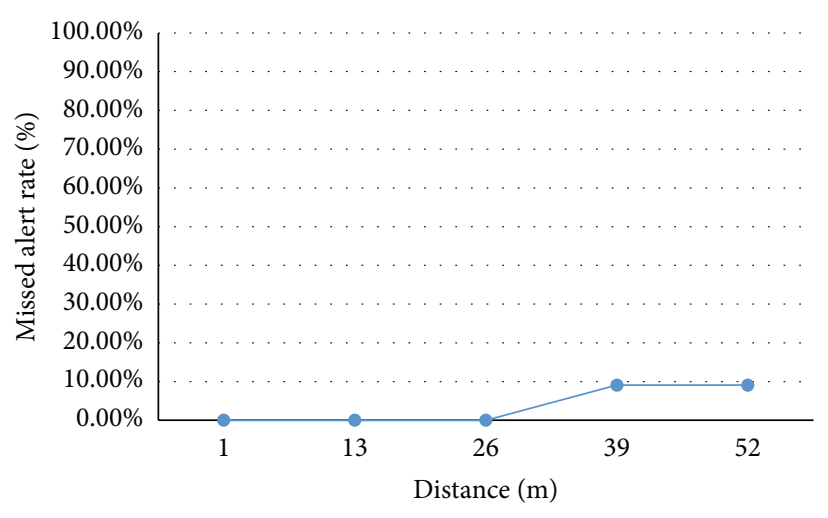

(b)

Figure 12: Results of alert latency versus distance (a). Missed alerts versus distance (b).

warning device varied from 1 meter to 52 meters. For each of these distances, several measurements were performed, and the average and standard deviation of the latency were calculated. These results are shown in Figure 12. Due to the dynamic polling period based on the wireless reception quality, the alert latency does not present a significant average change in relation to the distance. However, the standard deviation is more irregular since the needed network poll events to receive an alarm may be greater at longer distances. In distances up to $40 \mathrm{~m}$, all danger alerts were correctly delivered, but, when the warning device is farther away from a sensor node, the possibility of not receiving an alarm is not negligible. As for the higher standard deviation on the 1meter test, it is caused by the longer poll period since wireless reception at that distance is almost perfect. A long poll period increases deviation due to the higher possible time between a detection and the next poll.

On another test, the false positive rate of our system was measured. This test consisted in counting the times our system triggered an alarm in a case where it should not have. In order to do this, we set up a sensor node with its virtual detection line parallel to the traffic direction, in the same position it would be when forming part of a cone barrier closing a lane. This experiment setup is shown in Figure 13. Under these conditions, and with traffic following its expected direction, no alarm should be triggered.

The specific conditions and results of this experiment are presented in Table 4.

The results of this test show two false alarms out of a sample of 57 vehicles, a false positive rate of $3.5 \%$. The reason for these false positives can be attributed to the effect of wind on ultrasonic detectors, since the test run was carried out on a relatively windy day. Some authors have documented [13] that wind can cause erroneous readings in ultrasonic distance sensors, because it can induce disturbances on the emitted and reflected waves.

The last series of tests was aimed at evaluating the effect of impacts on the sensor node. The collision between a vehicle and a cone attached to a sensor node was not evaluated under real conditions, because it would have required the destructive test of several sensor nodes which were not available.
TABLE 4: False alarm test conditions.

\begin{tabular}{lc}
\hline Test duration & 15 minutes \\
\hline Average vehicle speed $(\bar{v})$ & $50-60 \mathrm{~km} / \mathrm{h}$ \\
\hline Ambient temperature & $17^{\circ} \mathrm{C}$ \\
\hline Vehicle count & 55 cars \\
& 2 buses \\
\hline Distance from vehicles $(d)$ & $1-1.5 \mathrm{~m}$ \\
\hline \multirow{2}{*}{ Results } & 1 false alarm triggered with an \\
& adjacent vehicle \\
& 1 false alarm triggered without an \\
& adjacent vehicle \\
\hline
\end{tabular}

However, a lab test was performed to assess the ability of the node to detect moderate impacts and cone tipping. No undetected events were reported on these tests, although it was found that, sometimes, the alarm is triggered by the ultrasonic range sensor in addition to the accelerometer, providing a welcome redundancy to the impact detection capability of the node. The reason for this is that, on its way down, the ultrasonic sensor can easily detect the ground closer than its predefined distance, treating this as a regular intrusion event.

\section{Conclusions}

Road maintenance and repair actuations often create a hazardous environment for both workers and drivers. Generally, these work zones are simply delimited by cones and coexist with regular traffic, so a distracted driver can enter the perimeter and provoke an extremely dangerous situation. In the past decades, several systems have been proposed to address this particular issue, although reviews and surveys agree on their limitations and defects.

In this paper, we propose and describe an innovative intrusion alarm system aimed at improving safety on these scenarios. Our solution consists of two main elements: sensor nodes based on ultrasonic beams to detect breaches in the perimeter and individual warning devices worn by 


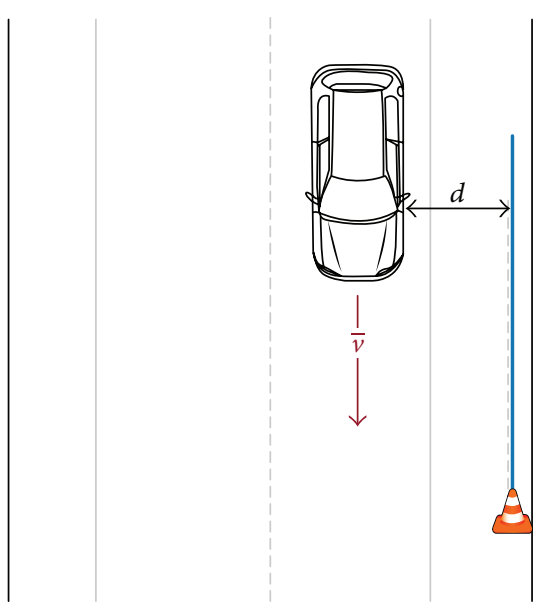

FIgURE 13: False alarm test setup.

the workers. All the elements in our system have wireless connectivity and are deployed forming a WSN that covers the whole work zone. This network effectively coordinates the operation of the system and is in charge of transmitting the alarms.

We have evaluated the performance of our system in a series of tests carried out under real conditions. The results of these tests confirm the effectiveness and usefulness of our solution and its suitability to the target scenario.

The inclusion of the WSN is the most innovative and substantial contribution of our solution. In addition to its current role, it presents a huge potential for additional applications which we will investigate in future works. For instance, individual working conditions could be monitored by means of the warning devices.

\section{Competing Interests}

The authors declare that there are no competing interests.

\section{Acknowledgments}

This work was partially funded by the company Valoriza Conservación de Infraestructuras and the Spanish Ministry of Industry, Energy, and Tourism through the Strategic Action on Economy and Digital Society (AEESD) under DEPERITA: TSI-100503-2015-39 Project.

\section{References}

[1] M. Wang, S. D. Schrock, Y. Bai, and R. Rescot, "Evaluation of innovative traffic safety devices at short-term work zones," Report K-TRAN: KU-09-5, The University of Kansas, 2011.

[2] G. Burkett, V. Her, and S. Velinsky, "Development of new kinds of mobile safety barriers," Final Report, AHCMT UC Davis, 2009.

[3] P. J. Kozdon, "Pulsed microwave motion sensor for intrusion detection,” US Patent 4,322,722, 8 pages, 1982.
[4] C. Nelson and R. E. Bos, "Roadway incursion alert system," US Patent 7,030,777, Logic Systems Inc., Sacramento, Calif, USA, 2006.

[5] M. Tubaishat, P. Zhuang, Q. Qi, and Y. Sang, "Wireless sensor networks in intelligent transportation systems," Wireless Communications and Mobile Computing, vol. 9, no. 3, pp. 287-302, 2009.

[6] A. Pascale, M. Nicoli, F. Deflorio, B. Dalla Chiara, and U. Spagnolini, "Wireless sensor networks for traffic management and road safety," IET Intelligent Transport Systems, vol. 6, no. 1, pp. 67-77, 2012.

[7] K. Aziz, S. Tarapiah, M. Alsaedi, S. H. Haj, and S. Atalla, "Wireless sensor networks for road traffic monitoring," International Journal of Advanced Computer Science and Applications, vol. 6, no. 11, 2015.

[8] T. M. Hussain, A. M. Baig, T. N. Saadawi, and S. A. Ahmed, "Infrared pyroelectric sensor for detection of vehicular traffic using digital signal processing techniques," IEEE Transactions on Vehicular Technology, vol. 44, no. 3, pp. 683-689, 1995.

[9] Y. Jo, J. Choi, and I. Jung, "Traffic information acquisition system with ultrasonic sensors in wireless sensor networks," International Journal of Distributed Sensor Networks, vol. 2014, Article ID 961073, 12 pages, 2014.

[10] A. Haoui, R. Kavaler, and P. Varaiya, "Wireless magnetic sensors for traffic surveillance," Transportation Research Part C: Emerging Technologies, vol. 16, no. 3, pp. 294-306, 2008.

[11] R. Wang, L. Zhang, R. Sun, J. Gong, and L. Cui, "EasiTia: a pervasive traffic information acquisition system based on wireless sensor networks," IEEE Transactions on Intelligent Transportation Systems, vol. 12, no. 2, pp. 615-621, 2011.

[12] M. Bathula, M. Ramezanali, I. Pradhan, N. Patel, J. Gotschall, and N. Sridhar, "A sensor network system for measuring traffic in short-term construction work zones," in Distributed Computing in Sensor Systems: 5th IEEE International Conference, DCOSS 2009, Marina del Rey, CA, USA, June 8-10, 2009. Proceedings, vol. 5516 of Lecture Notes in Computer Science, pp. 216-230, Springer, Berlin, Germany, 2009.

[13] L. Alonso, V. Milanés, C. Torre-Ferrero, J. Godoy, J. P. Oria, and T. De Pedro, "Ultrasonic sensors in urban traffic driving-aid systems," Sensors, vol. 11, no. 1, pp. 661-673, 2011. 


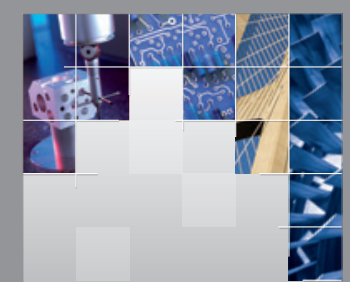

\section{Enfincering}
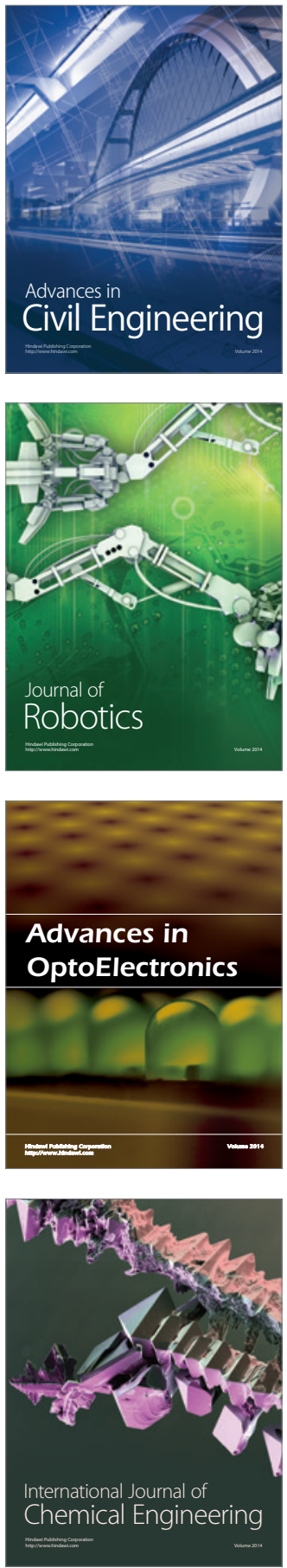

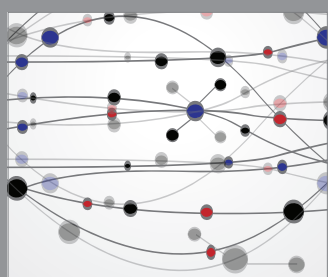

The Scientific World Journal

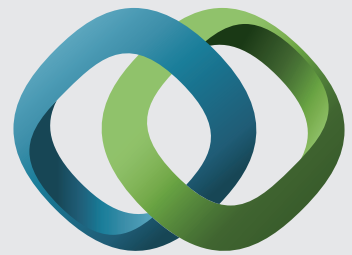

\section{Hindawi}

Submit your manuscripts at

http://www.hindawi.com
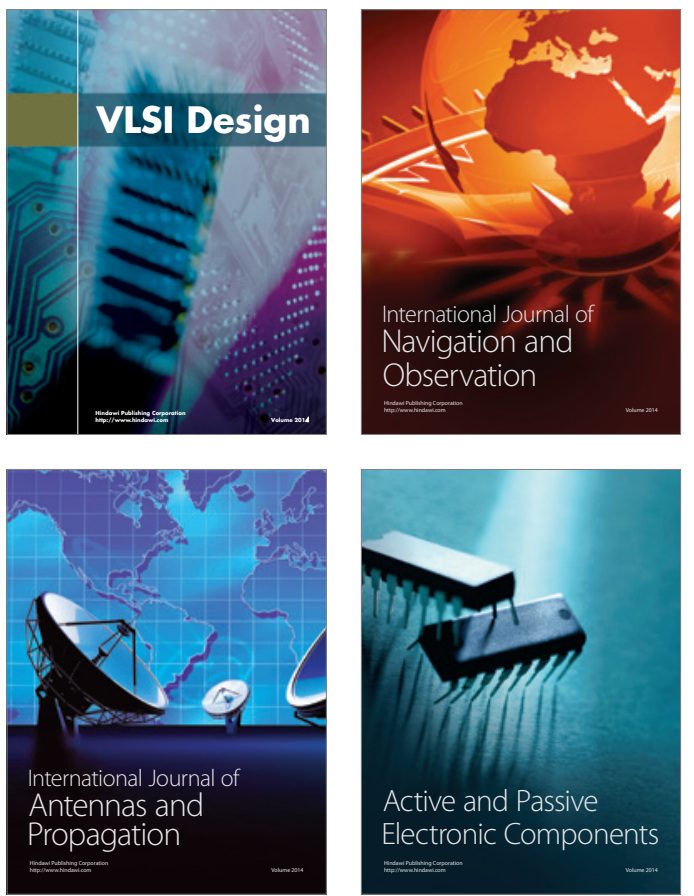
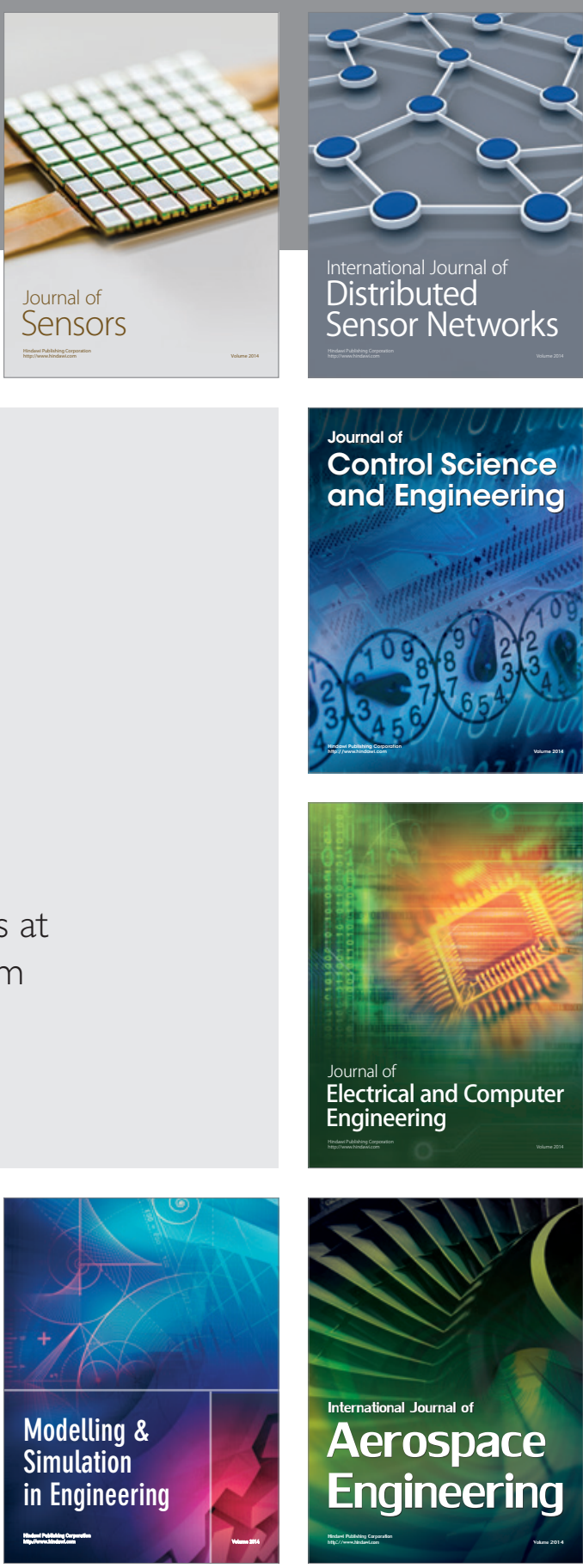

International Journal of

Distributed

Sensor Networks

Journal of

Control Science

and Engineering
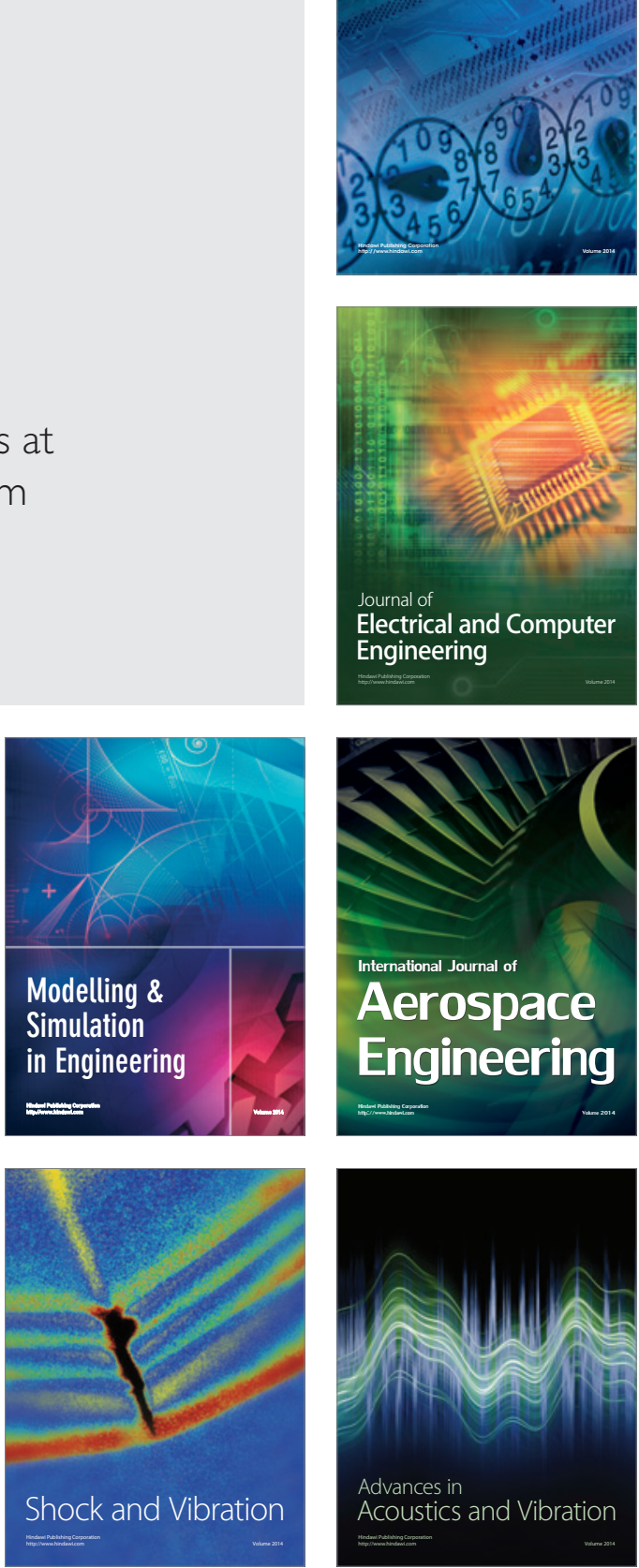